\title{
Support Vector Networks for Prediction of Floor Pressures in Shallow Cavity Flows
}

\author{
Mehmet Önder Efe, Marco Debiasi, Peng Yan, Hitay Özbay and Mohammad Samimy
}

\begin{abstract}
During the last decade, Support Vector Machines (SVM) have proved to be very successful tools for classification and regression problems. The representational performance of this type of networks is studied on a cavity flow facility developed to investigate the characteristics of aerodynamic flows at various Mach numbers. Several test conditions have been experimented to collect a set of data, which is in the form of pressure readings from particular points in the test section. The goal is to develop a SVM based model that emulates the one step ahead behavior of the flow measurement at the cavity floor. The SVM based model is built for a very limited amount of training data and the model is tested for an extended set of test conditions. A relative error is defined to measure the reconstruction performance, and the peak value of the FFT magnitude of the error is measured. The results indicate that the SVM based model is capable of matching the experimental data satisfactorily over the conditions that are close to the training data collection conditions, and the performance degrades as the Mach number gets away from the conditions considered during training.
\end{abstract}

\section{INTRODUCTION}

It is a well known fact that skin friction on air vehicles reduces the maneuverability and agility while increasing the fuel consumption. Material fatigue and damage to stores are other related problems that point the pursuit of techniques for reducing the skin friction. One alternative towards this goal is the active control of near body aerodynamic behavior. The practical significance of this work is on the model development side of the above mentioned ultimate goal.

Feedback control performance on aerodynamic flow systems heavily depends upon the capabilities of a representative model. The process under investigation nonlinear and the governing dynamics is described by Navier-Stokes equations, which display quite complicated behavior in aerodynamic

This work was supported in part by AFRL/VA and AFOSR under contract no F33615-01-2-3154 and in part by the European Commission under contract no. MIRG-CT-2004-006666 and in part by TOBB ETÜ, BAP Program, under contract no ETÜ-BAP-2006/04

Dr. Efe is the corresponding author, and he is with TOBB Economics and Technology University, Department of Electrical and Electronics Engineering, Sögütözü, Ankara, Turkey, Phone: +90-312-292-4064, Fax: +90312-292 4091 onderefedieee.org

Dr. Debiasi is with the Department of Mechanical Engineering, The Ohio State University, Columbus, OH 43210, U.S.A. debiasi . 1 @osu . edu

Dr. Yan was with Department of Electrical and Computer Engineering, The Ohio State University, Columbus, OH 43210, USA; present address: Seagate Tech Center, Bloomington MN 55435 U.S.A. yan.39@osu.edu

Dr. Özbay was with the Department of Electrical and Electronics Engineering, Bilkent University, Bilkent, TR-06800, Ankara, Turkey, on leave from Department of Electrical and Computer Eng., The Ohio State University hitay@bilkent.edu.tr, ozbay@ece.osu.edu

Dr. Samimy is with the Department of Mechanical Engineering, The Ohio State University, Columbus, OH 43210, U.S.A. samimy. 1 @osu. edu flows and this entails high sampling rates. The described nature of the problem highlights how substantial the performance at the modeling stage is. Modeling of such a process using machine learning methods is one alternative that is motivated also by the facts that real-time observations are generally noise corrupted and even rough models of the overall system constituents such as actuators, sensors and system dynamics are unavailable. From an input-output data processing point of view, the problem in hand is a good test bed where machine learning algorithms can be applied. This paper focuses on an increasingly popular tool named Support Vector Machines.

In the past years, several soft computing tools have been used for modeling of aerodynamic systems, for example Neural Networks (NN) in [1-7], fuzzy logic in [8-11] and SVM in [12].

In [1], Jacobson and Reynolds conducted a numerical study on the control of wall shear stress in a boundary layer by using feedforward NN as inverse controllers, which showed skin friction reduction by about $8 \%$. The study of active laminar flow control [2] showed that a properly trained NN can establish complex nonlinear relationships between multiple inputs and outputs which are peculiar to an active flow control system. The work demonstrates the cancellation of wave disturbances in transitional boundary layers by a pretrained neural models. Sensors measure either wall pressure or wall shear stress. Training strategies and performance measures are considered, and fault tolerance capability of NN is emphasized. Faller et al., [3], obtained a NN model of a pitching airfoil based on experimental data. With limited training data, the model predicts unsteady surface pressure topologies within $5 \%$ of what is available in the experimental data. Given the actuator control signals, the NN anticipates the interactions between the unsteady flow field and airfoil. The NN has a very complex structure configuration. Gradient descent is used for training and the pressure values on the airfoil are estimated by using the recordings of angle of attack and its time derivative. The NN controller has 6-12-12-1 configuration, and a desired lift/drag response is aimed to be observed. It is possible to extend the results focusing on $\mathrm{NN}$ use in aerodynamic system modeling (See [4-7] and the references therein).

As another alternative, Fuzzy Logic (FL) is a practical framework for solving complicated problems by utilizing expert knowledge. The practicality of the paradigm stems from the fact that the human expertise is expressed in the form of IF antecedent THEN consequent statements, i.e. the task to be achieved is modeled through the use of linguistic 
descriptions. In [8], Cohen et al. use FL for the control of a circular cylinder vortex shedding model. The fuzzy system in [8] has been used to scale a control signal produced by a PID controller, and it has been shown that such a strategy yields significant improvement in the performance compared to the sole PID solution. In [9], FL with triangular membership functions is used for controlling the vortex flows on a generic X29-A model. The fuzzy controller is compared with neural controllers and predictive schemes. Dragojlovic et al. utilize the fuzzy logic in improving the performance of a Computational Fluid Dynamics (CFD) solver. The fuzzy control scheme guides the increment in the relaxation factor by using triangular membership functions, [10-11]. Depending on the past solution entries, the CFD solver automatically adjusts itself to exploit the best relaxation factor.

In 1995, Vapnik proposed a new approach for classification and regression problems, named support vector machines, [13]. This new approach aims at minimizing the structural risk, i.e. the upper bound of the generalization error. By this means, SVMs are superior to conventional NN, the training algorithms of which minimize the empirical risk over a set of training pairs, [14-15]. In [12], five hole pressure probe calibration is studied comparatively with NN models and it is seen that SVM predictions are much better than those obtained with NN trained under the same operating conditions. In the same paper, it is shown that utilizing the SVMs, efficiency of the response surface technique can be increased for CFD based shape optimizations. As the test bed, diffusers converting the the dynamic pressure to static pressure rise is chosen and response surface has been constructed with the aid of SVM. In [16], Gretton et al., present a SVM based identification of a robot arm and the regressor used in [16] has a similar structure as we use in this paper.

As outlined above, some work has been done in the past decade to explore the use of machine learning techniques in flow modeling and control with various degrees of success. Several of these works showed promising results but were based on numerical simulations and lacked any experimental validation of the concept. The few experimental studies available are concerned with slowly varying states of the flow. To the best of our knowledge, no attempt has been made so far in using SVM to model a more dynamic, higher frequency flow like the one over a cavity. Therefore, many questions remain open about the merit and effectiveness of the tools exploiting statistical learning theory in flow modeling and control. Having this motivation in mind, in this paper, we work on the experimental setup shown in Figs. 1-2 and introduced in the next section. The goal is to characterize the flow passing over a cavity based on surface pressure measurements. The third section summarizes the modeling based on SVM. The obtained simulation results are discussed in the fourth section, and conclusions constitute the last part of the paper.

\section{THE EXPERIMENTAL FACILITY}

In this study, the experimental facility described in more detail in [17-18] is used. The core of the experimental setup consists of an optically accessible, blow-down type wind tunnel with a test section of equal width and height, $W=H=50.8 \mathrm{~mm}$. A cavity that spans the entire width of the test section is recessed in the floor with a depth $D=12.7 \mathrm{~mm}$ and length $L=50.8 \mathrm{~mm}$ for an aspect ratio $L / D=4$. For control, the cavity shear-layer is forced by a 2-D synthetic-jet type actuator issuing from the end slot of a high-aspect-ratio converging nozzle embedded in the cavity leading edge and spanning the width of the cavity, see Fig. 2. Actuation is provided by the movement of the titanium diaphragm of a Selenium D3300Ti compression driver whose input signal is amplified by a Crown D-150A amplifier. The pressure fluctuations are measured by Kulite dynamic pressure transducers placed in different locations in the test section, see Fig. 3.

Since the experimental facility enables us to acquire pointwise observations from the critical locations of the cavity, one could use this information for estimation of the flow inside the cavity. This is done using a dSPACE 1103 DSP board connected to a Dell Precision Workstation 650. This system acquires the pressure transducer signals simultaneously at a sampling frequency of $50 \mathrm{kHz}$ through 16-bit input channels, and manipulates them to produce the desired output signal from a 14-bit output channel. Each recording is band-pass filtered between $200 \mathrm{~Hz}$ and $10 \mathrm{kHz}$ to remove spurious frequency components. The simultaneous time traces collected from these transducers have been used to train the support vector network with the characteristics described in [19-21]. It is critically important to emphasize that the data must be spectrally rich enough to capture cases that are likely to be encountered in real-time operation. This makes sure that the $\mathrm{NN}$ responds appropriately to the input variables.

In [18], it is observed that the cavity flow exhibits strong, single-mode resonance in the Mach number ranges 0.25-0.31 and 0.39-0.5, and multi-mode resonance in the Mach number range $0.32-0.38$. In the same study, it is observed that the frequency of sinusoidal forcing with the synthetic jet-like actuator has a major impact on the cavity flow resonance whereas the effect of the amplitude is relatively minor and it affects the control authority only at higher Mach numbers. This prompted the development of a logic-based type of control that searches the forcing frequencies in a closed-loop fashion that reduce the cavity flow resonant peaks and then maintains the system in such conditions through an openloop control. The technique performed well in the experimental trials and allowed identification of optimal frequencies for the reduction of resonant peaks in the Mach number range $0.25-0.5$. Another indication of this result was the adequate control authority introduced by the actuators. Some effort within the described research has been dedicated to design classical controllers and these succeeded to some extent. The experience gained during these trials have stipulated that 


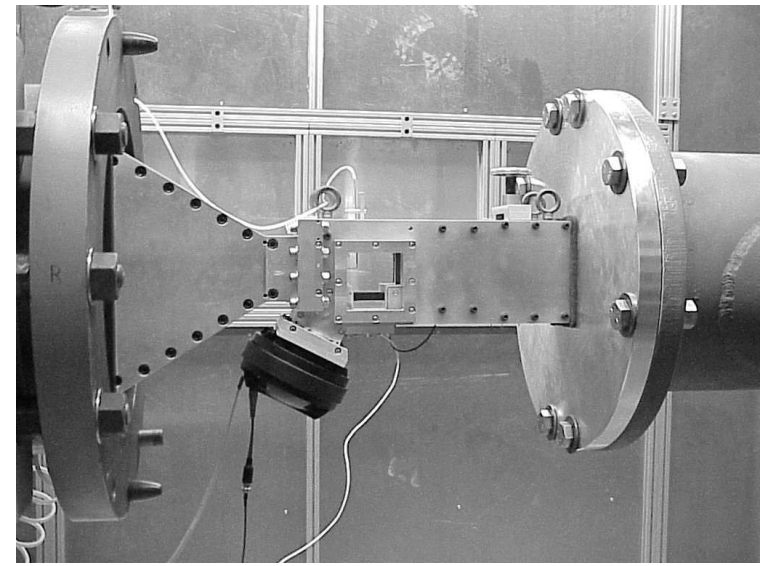

Fig. 1. A photograph of the cavity flow facility

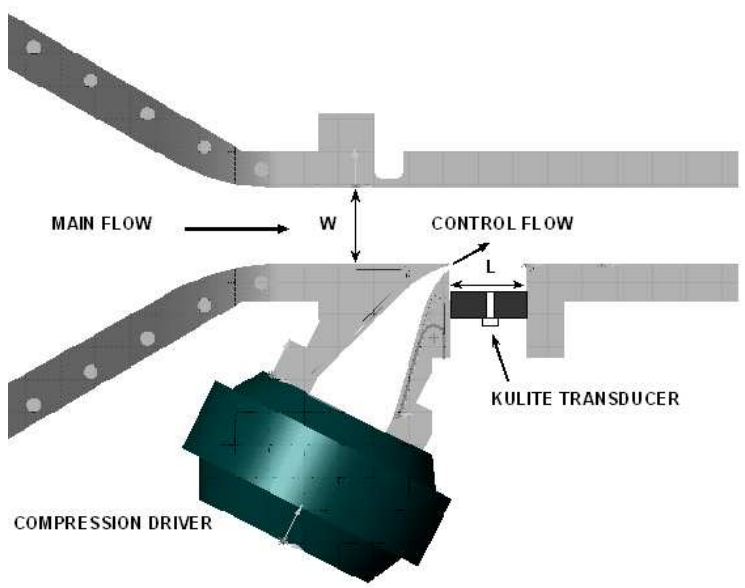

Fig. 2. Cutout of the wind tunnel showing the converging nozzle, the test section, the cavity, the actuator coupling, and the placement of a Kulite transducer in the cavity floor

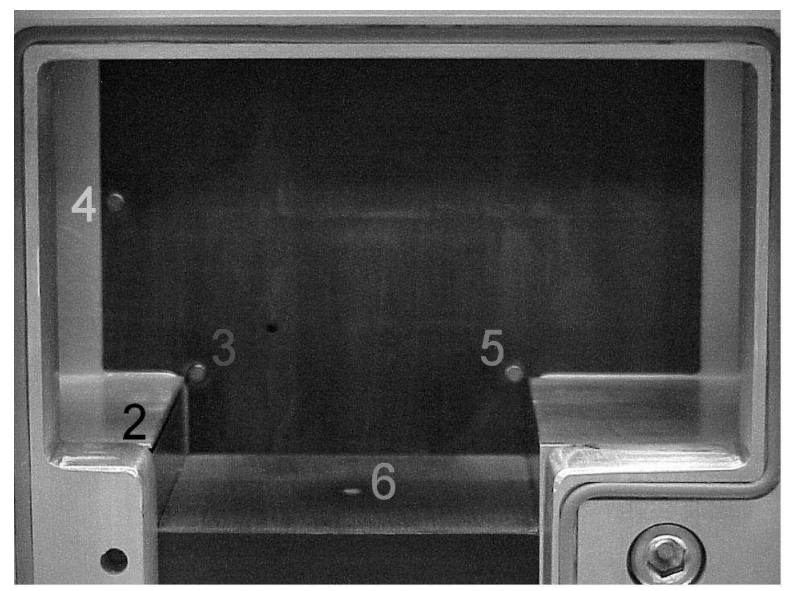

Fig. 3. The locations of the pressure transducers placed in the test section the modeling of the process deserves particular attention as the desired closed loop control performance depends strictly upon the representational capability of the process model. Since the experimental facility enables us to acquire pointwise observations from the physically critical locations of the cavity, one could use this information for identification of the cavity flow and this paper discusses how SVMs could be utilized for this purpose.

\section{Modeling By Support Vector Machines}

A. SVM Basics

Consider the regression problem over the pairs

$$
\mathcal{D}=\left\{\left(\mathbf{u}_{1}, d_{1}\right), \ldots,\left(\mathbf{u}_{N}, d_{N}\right)\right\}, \quad \mathbf{u}_{i} \in \mathbb{R}^{m}, \quad d \in \mathbb{R}
$$

with a function

$$
f(\mathbf{u})=\langle\mathbf{w}, \mathbf{u}\rangle+b
$$

where $\mathbf{w}$ and $b$ denote the weight vector and the bias value, respectively. $\langle\cdot, \cdot\rangle$ stands for an appropriately defined operator, which is an inner product for linear regression and a kernel for nonlinear regression. Defining a quadratic loss function as in (3) quantifies the performance for the $i$ th pair,

$$
L\left(d_{i}, f\left(\mathbf{u}_{i}\right)\right)=\left(d_{i}-f\left(\mathbf{u}_{i}\right)\right)^{2} .
$$

Minimizing the empirical risk given by (4) lets us obtain the best values of $\mathbf{w}_{i}$ s causing least complexity represented by $\|\mathbf{w}\|^{2}$

$$
R=\frac{1}{2}\|\mathbf{w}\|^{2}+C \sum_{i=1}^{N} L\left(d_{i}, f\left(\mathbf{u}_{i}\right)\right)^{2},
$$

where $C$ is a parameter determining the relative importance of the terms contributing to $R,[14]$. The primal form of the optimization problem can be expressed compactly as

$$
\begin{gathered}
\min _{\mathbf{w}, b} \frac{1}{2}\|\mathbf{w}\|^{2}+C \sum_{i=1}^{N}\left(\zeta_{i}^{2}+\hat{\zeta}_{i}^{2}\right) \\
\text { s.t. }\left\{\begin{array}{c}
f\left(\mathbf{u}_{j}\right)-d_{j} \leq \zeta_{j}, \\
d_{j}-f\left(\mathbf{u}_{j}\right) \leq \hat{\zeta}_{j}, \quad, \quad j=1,2, \ldots, N \\
\zeta_{j}, \hat{\zeta}_{j} \geq 0
\end{array}\right.
\end{gathered}
$$

where $\zeta_{j}$ and $\hat{\zeta}_{j}$ are slack variables penalizing the deviations from the target output. The above described problem can be converted into a convex quadratic optimization problem by writing the dual representation. The solution can be obtained by introducing the Lagrange multipliers and performing the following minimization for $\beta \in \mathbb{R}^{N}$;

$$
\min _{\beta} \frac{1}{2} \sum_{i=1}^{N} \sum_{j=1}^{N} \beta_{i} \beta_{j}\left\langle\mathbf{u}_{i}, \mathbf{u}_{j}\right\rangle-\sum_{i=1}^{N} \beta_{i} d_{i}+\frac{1}{2 C} \sum_{i=1}^{N} \beta_{i}^{2},
$$

with constraint $\sum_{i=1}^{N} \beta_{i}=0$. It should be noted that the support vectors are the $u_{i}$ s for which the corresponding $\beta_{i}$ is nonzero. The result of the minimization lets us obtain 


$$
\begin{gathered}
\mathbf{w}^{*}=\sum_{i=1}^{N} \beta_{i} \mathbf{u}_{i}, \\
b^{*}=\frac{1}{N} \sum_{i=1}^{N}\left(y_{i}-\sum_{j=1}^{N}\left\langle\mathbf{u}_{i}, \mathbf{u}_{j}\right\rangle \beta_{j}\right)
\end{gathered}
$$

which are to be used in (2). The nonlinear regression problem is to replace the operator $\langle\cdot, \cdot\rangle$ in (1) with a kernel function satisfying the Mercer conditions, [15].

\section{B. Application of SVM for Aerodynamic Flow Modeling}

In the training phase, the SVM based model is asked to realize the mapping from current state of the flow and external excitation to the next state of the flow. The state of the flow is described by the information acquired from the chosen sensors. According to Figs. 2-3, the sensor labelled $S_{1}$ measures $u_{1, k}$, the actuation signal at time $k$ in Volts, $S_{2}$ measures $u_{2, k}$, the pressure fluctuations just before the actuator exit, $S_{3}$ measures $u_{3, k}$, the pressure fluctuation just after the actuator exit (i.e. at the shear layer receptivity region just downstream of the cavity leading edge), $S_{4}$ measures $u_{4, k}$, the pressure fluctuations (if any) before the cavity, $S_{5}$ measures $u_{5, k}$, the pressure fluctuations at the cavity trailing edge, $S_{6}$ measures $d_{k}$, the pressure fluctuations at the center of the cavity floor. The signals from these transducers are simultaneously sampled with the host computer.

With these definitions, a series-parallel SVM based emulator is desired to match the training data in (1). It should be noted that the input vector is composed of the information coming from the above sensors and their delayed (past) values, whose delay depths are specified by the designer. Notice that the Mach number could also be an external input to the SVM model to characterize the dynamical composition of various experimental regimes within a single support vector network. If such an approach succeeds, we obtain a SVM emulator that can be used at Mach numbers around Mach $=0.30$ regime. Towards this goal, we have collected a set of experimental data for several test cases as tabulated in Table I.

TABLE I

DATA ACQUisition CONDITIONS FOR TRAINING SET

\begin{tabular}{|c|c|c|}
\hline Mach Number & Excitation Frequency & Excitation Magnitude \\
\hline \hline 0.28 & $3250 \mathrm{~Hz}$ & $2.35 \mathrm{Vrms}$ \\
0.28 & $3920 \mathrm{~Hz}$ & $4.06 \mathrm{Vrms}$ \\
0.32 & $3250 \mathrm{~Hz}$ & $2.35 \mathrm{Vrms}$ \\
0.32 & $3920 \mathrm{~Hz}$ & $4.06 \mathrm{Vrms}$ \\
\hline
\end{tabular}

Every experiment shown above contributes only 126 samples to the training data set, which excludes Mach $=0.30$ case. This is a deliberate choice for test data as Mach $=0.30$ displays quite rich spectral view making the corresponding phenomenon difficult to model compactly. The total number of training samples is 504, which provides clearly very limited information to perform a satisfactory modeling. One might prefer to enlarge the training data set to cover a richer set of cases yet the cost of this is a significant increase in the training time.

At discrete time index $k$, the input vector to the SVM is as given below

$$
\mathbf{u}_{k}=\left(u_{1, k}, u_{6, k}, u_{6, k-1}, u_{3, k}, u_{5, k}, \text { Mach }\right)
$$

The desired output for this input pattern is $d_{k}=u_{6, k+1}$. In order to validate the modeling claim of the paper, the mechanism in (1) is implemented with the discussed SVM structure having 6 inputs, and one output. The training has been achieved by using the software available at http://www.isis.ecs.soton.ac.uk, [14]. The rationale that lies behind is the minimization of the discrepancy between the process outputs and the SVM model response over a set of input-output pairs while maintaining the minimal structural risk. A linear kernel is utilized, i.e. kernelized value of $u_{k}$ and $u_{l}$ is $\left\langle u_{k}, u_{l}\right\rangle=u_{k} u_{l}^{\mathrm{T}}$ and the SVM model is obtained approximately after a 2.3 hours of training process.

In Fig.4, the validation of the obtained SVM model is shown for one of the unseen operating conditions (in terms of Mach number), which correspond to the case described by Mach $=0.30$. In this figure, $d_{k}$ and $x_{k}$ denote the desired (already recorded) value and prediction of SVM based model $x_{k+1}=f\left(u_{k}\right)$, respectively. The obtained results are reasonably good to claim that the model functions well for the considered operating conditions. We can quantify this by defining the relative error $e_{\text {rel }}$ as the ratio of the average powers of $d$ and $d-x$ over the time interval $t \in\left[0, T_{f}\right]$, where $T_{f}=163.84 \mathrm{msec}$, that is

$$
e_{\text {rel }}:=\frac{\frac{1}{T_{f}} \int_{0}^{T_{f}}|d(t)-x(t)|^{2} \mathrm{~d} t}{\frac{1}{T_{f}} \int_{0}^{T_{f}}|d(t)|^{2} \mathrm{~d} t} .
$$

The numerical results presented in Fig. 4, give $e_{\text {rel }}=$ 0.0431 , i.e. average power of the error signal $d(t)-x(t)$ is $4.31 \%$ of the average power of the signal $d(t)$. Clearly from (11), the smaller the $e_{\text {rel }}$ the better the reconstruction performance. To sum up, when looking at the result illustrated in Fig. 4, the similarity of the desired and estimated signals is found to be promising.

Although the similarity in time domain is one way of demonstrating the performance we need to check the spectral views to strengthen the theoretical claims. In Fig. 5, we demonstrate the Fast Fourier Transform (FFT) of the signals involved in the procedure. The upper subplot depicts the FFT magnitudes of $d(t)$ and $x(t)$ over the $200 \mathrm{~Hz}-10 \mathrm{kHz}$ band of the spectrum. Obtaining a similarity over this range of frequencies is sufficient as the important information is present in this band. The lower subplot illustrates the FFT magnitude of the difference $d(t)-x(t)$. The resonant peak is visible in both subplots and the peak value in the lower subplot, which is reasonably small, emphasizes that the phase of the prediction reasonably fits the desired signal.

In Fig. 6, we zoom the behavior in the vicinity of the peak at $3920 \mathrm{~Hz}$. The two FFT magnitude plots are very close 
$d_{k}$ (Desired, red) \& $x_{k}$ (SVM, black) signals $d_{k}$ (Desired, red) \& $x_{k}$ (SVM, black) signals
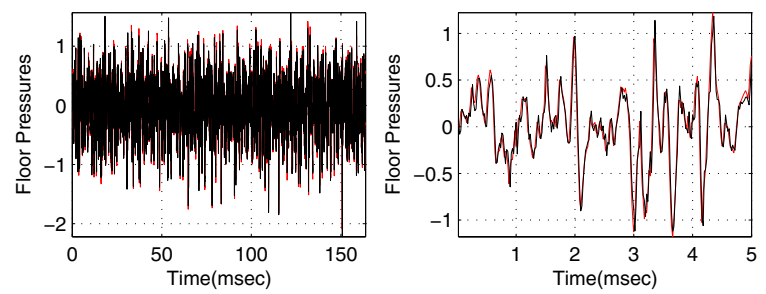

Difference Between $d_{k}$ and $x_{k}$

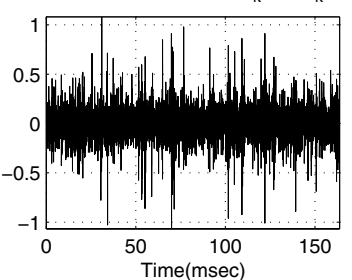

Difference Between $d_{k}$ and $x_{k}$

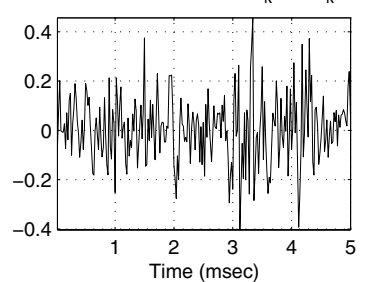

Fig. 4. The time view of the signals and the error for Mach 0.3 . The excitation signal is a sinusoidal signal having frequency $3920 \mathrm{~Hz}$ and magnitude $4.06 \mathrm{~V}$ rms
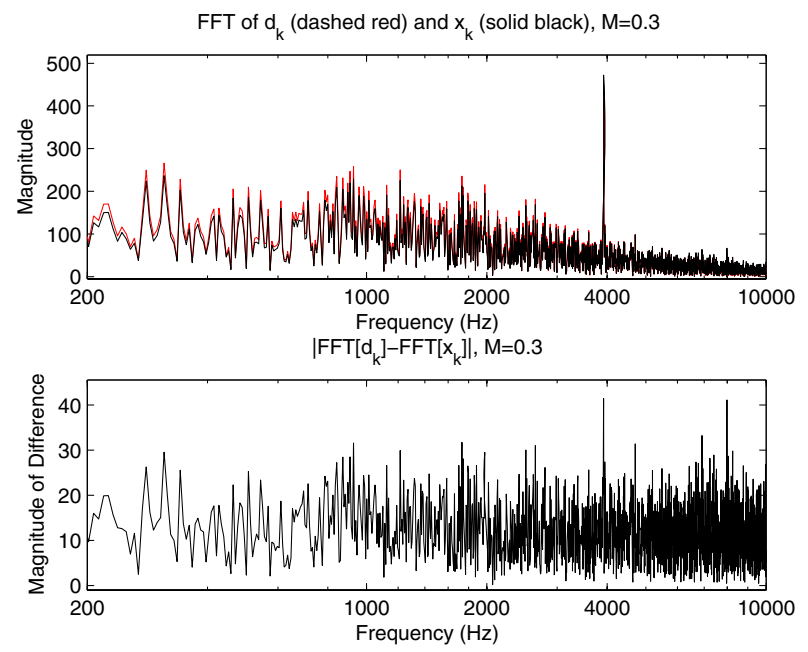

Fig. 5. Spectral view of the signals and the error for Mach 0.3. The excitation signal is a sinusoidal signal having frequency $3920 \mathrm{~Hz}$ and magnitude $4.06 V_{\mathrm{rms}}$

to each other, which stipulate that the SVM based model performs well under the depicted operating conditions.

In Table II, we summarize the results for an extended set of operating conditions including the one above. In each case, we compute the relative error in (11) and the peak value of the FFT magnitude of the error $d(t)-x(t)$. We consider Mach numbers $0.25,0.28,0.30,0.32$ and 0.35 . For each of these cases, we perform three sets of experiments. First experiment set comprises the noise driven cases, i.e. the actuator is excited by a noise signal within the allowed physical limits of the actuator. The second set of experiments provides data for the excitation by a sinusoidal signal having frequency $f_{\text {exc }}=3250 \mathrm{~Hz}$ and amplitude $A_{\text {exc }}=2.35 \mathrm{~V}_{\mathrm{rms}}$. The cases in the third experiment set is similar to those in the second one but with a sinusoidal signal having frequency
$3920 \mathrm{~Hz}$ and amplitude $4.06 \mathrm{~V}_{\mathrm{rms}}$. In all these cases the highest value of $e_{\text {rel }}$ turns out to be less than 0.065 .
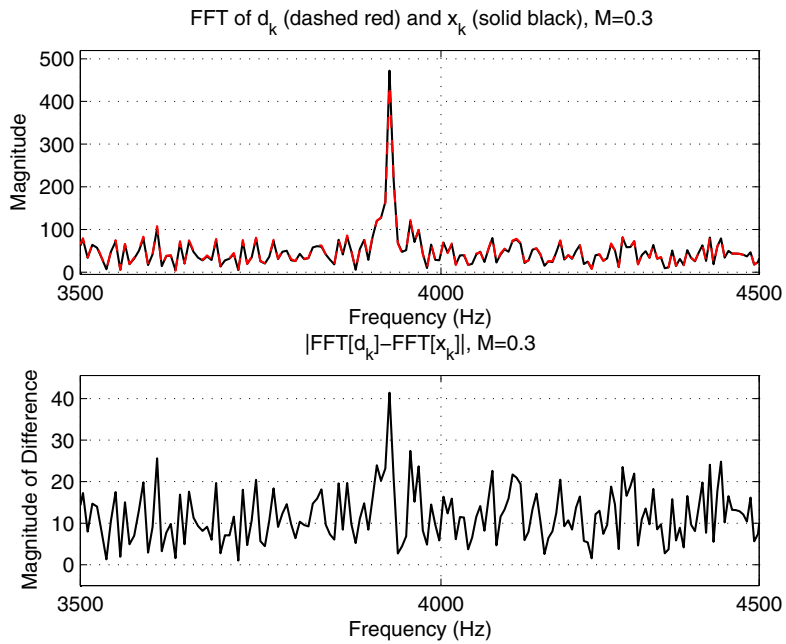

Fig. 6. Zoomed spectral view of the signals and the error for Mach 0.3 The excitation signal is a sinusoidal signal having frequency $3920 \mathrm{~Hz}$ and magnitude $4.06 V_{\mathrm{rms}}$

TABLE II

The Operating Conditions For VALIDATING THE SVM BASED MODEL

\begin{tabular}{|c|c|c|c|c|}
\hline Mach & $f_{\text {exc }}$ & $A_{\text {exc }}$ & $e_{\text {rel }}$ & sup $|\mathrm{FFT}(d-x)|$ \\
\hline \hline 0.25 & Noise & Below sat. & 0.048475 & 20.9356 \\
0.28 & Noise & Below sat. & 0.041880 & 44.7202 \\
0.30 & Noise & Below sat. & 0.045057 & 51.3371 \\
0.32 & Noise & Below sat. & 0.047126 & 44.6895 \\
0.35 & Noise & Below sat. & 0.054596 & 57.8424 \\
0.25 & $3250 \mathrm{~Hz}$. & $2.35 \mathrm{~V}_{\text {rms }}$ & 0.042515 & 78.3571 \\
0.25 & $3920 \mathrm{~Hz}$. & $4.06 \mathrm{~V}_{\text {rms }}$ & 0.044337 & 99.6471 \\
0.28 & $3250 \mathrm{~Hz}$. & $2.35 \mathrm{~V}_{\text {rms }}$ & 0.039993 & 78.5551 \\
0.28 & $3920 \mathrm{~Hz}$. & $4.06 \mathrm{~V}_{\text {rms }}$ & 0.047418 & 72.5547 \\
0.30 & $3250 \mathrm{~Hz}$. & $2.35 \mathrm{~V}_{\text {rms }}$ & 0.051279 & 69.4989 \\
0.30 & $3920 \mathrm{~Hz}$. & $4.06 \mathrm{~V}_{\text {rms }}$ & 0.043100 & 39.4168 \\
0.32 & $3250 \mathrm{~Hz}$. & $2.35 \mathrm{~V}_{\text {rms }}$ & 0.056608 & 65.0521 \\
0.32 & $3920 \mathrm{~Hz}$. & $4.06 \mathrm{~V}_{\text {rms }}$ & 0.054018 & 82.9277 \\
0.35 & $3250 \mathrm{~Hz}$. & $2.35 \mathrm{~V}_{\text {rms }}$ & 0.058321 & 151.9084 \\
0.35 & $3920 \mathrm{~Hz}$. & $4.06 \mathrm{~V}_{\text {rms }}$ & 0.064033 & 196.5640 \\
\hline
\end{tabular}

Comparing the results given in the rightmost column of Table II, one sees that the performance is satisfactory around Mach $=0.30$ and it gradually decreases as the Mach number gets away from 0.30. This result is an expected as the flow properties change dramatically as the Mach number changes and the devised SVM model maintains its validity only around Mach $=0.30$.

A brief comparison of SVM based approach presented here and that based on feedforward NNs differ significantly in terms of the number of samples entering the modeling procedure. SVM based models can be achieved with considerably smaller number of data sets as their tuning is based on the minimization of an upper bound on erors, while NNs are configured to minimize the error based on a set of given data. The reader is referred to [19], [20] for more details about the issues on $\mathrm{NN}$ based flow modeling. 


\section{CONCLUSIONS}

This paper focuses on the modeling issues for subsonic cavity flows. An experimental setup has been constructed for this purpose and the goal is to show that the surface pressure readings could lead to a SVM based model for predicting the behavior at the cavity floor. The results have demonstrated that the goal is attainable with a simple SVM structure admitting the Mach number as one of the input variables. This makes it possible to utilize the SVM over a range of regimes characterized by the Mach number. The results obtained through the conducted research advances the subject area to the development of models based on statistical learning theory which can effectively describe the flow dynamics. The very limited number of training samples and the accuracy in extracting the features deserve emphasis. Short term research goal aims to improve the training time of SVM based models to incorporate more input-output pairs into the regression problem while in the long term, the authors aim at finding the best representative model and best feedback controller closing the loop by meeting the performance specifications admissibly.

\section{Acknowledgments}

The authors would like to thank Dr. S. İplikçi, Dr. J.H. Myatt, Dr. J. DeBonis, Dr. R.C. Camphouse, X. Yuan, E. Caraballo, J. Malone and J. Little for fruitful discussions in devising the presented work.

\section{REFERENCES}

[1] Jacobson, S.A. and Reynolds, W.C., "Active Control of Boundary Layer Wall Shear Stress Using Self-Learning Neural Networks," AIAA Shear Flow Conferece, July 6-9, Orlando, FL, USA, 1993 (Paper No AIAA 93-3272).

[2] Fan, X., Hofmann, L. and Herbert, T., "Active Flow Control with Neural Networks," AIAA Shear Flow Conferece, July 6-9, Orlando, FL, USA, 1993 (Paper No AIAA 93-3273).

[3] Faller, W.E., Schreck, S.J. and Luttges, M.W., "Real-Time Prediction and Control of Three Dimensional Unsteady Separated Flow Fields Using Neural Networks," 32nd Aerospace Sciences Meeting and Exhibit, January 10-13, Reno, NV, USA, 1994 (Paper No AIAA 94-0532).

[4] Kawthar-Ali, M.H. and Acharya, M., "Artificial Neural Networks for Suppression of the Dynamic Stall Vortex over Pitching Airfoils," 34th Aerospace Sciences Meeting and Exhibit, January 15-18, Reno, NV, USA, 1996 (Paper No AIAA 96-0540).

[5] Lee, C., Kim, J., Babcock, D. and Goodman, R., "Application of Neural Networks to Turbulence Control for Drag Reduction," Physics of Fluids, v.9, no. 6, pp.1740-1747, 1997.

[6] Yuen, P.K. and Bau, H.H., "Controlling Chaotic Convection Using Neural Nets - Theory and Experiments," Neural Networks, v.11, pp.557, 1998.

[7] Giralt, F., Arenas, A., Ferre-Gine, J., Rallo, R. and Kopp, G.A. , “The Simulation and Interpretation of Free Turbulence with a Cognitive Neural System," Physics of Fluids, v.12, No. 7, pp.1826-1835, 2000.

[8] Cohen, K., Siegel, S. and McLaughlin, T. and Myatt, J.H., "Fuzzy Logic Control of a Circular Cylinder Vortex Shedding Model," 41th AIAA Aerospace Sciences Meeting, AIAA Paper No: 2003-1290, Reno, NV, U.S.A., 2003.

[9] Joshi, P. and Valasek, J., "Direct Comparison of Neural Network, Fuzzy Logic and Model Predictive Variable Structure Vortex Flow Controllers," Proceedings of the AIAA Guidance, Navigation and Control Conference, Portland, OR, 9-11 August 1999, (Paper No: AIAA-99-4279-CP)

[10] Dragojlovic, Z., Kaminski, D.A. and Ryoo, J., "Tuning of a Fuzzy Rule Set for Controlling Convergence of a CFD Solver in Turbulent Flow," International Journal of Heat and Mass Transfer, Vol.44, pp.3811-3822, 2001.
[11] Dragojlovic, Z. and Kaminski, D.A., "A Fuzzy Logic Algorithm for Acceleration of Convergence in Solving Turbulent Flow and Heat Transfer Problems," Numerical Heat Transfer, Part B, Vol.46, pp.301327, 2004.

[12] Fan, H.-Y., Dulikravich, G.S. and Han, Z.-X., "Aerodynamic Data Modeling Using Support Vector Machines," Inverse Problems in Science and Engineering, Vol. 13, No. 3, pp.261-278, 2005.

[13] Vapnik, V., The Nature of Statistical Learning Theory, New York, Springer-Verlag, 1995.

[14] Gunn, S.R., "Support Vector Machines for Classification and Regression,” ISIS Technical Report, University of Southampton, May 1998.

[15] Cristianini, N. and Shawe-Taylor, J., An Introduction to Support Vector Machines and Other Kernel-based Learning Methods, Cambridge University Press, 2000.

[16] Gretton, A., Doucet, A., Herbrich, R., Rayner, P.J.W. and Schölkopf, B., "Support vector regression for black-box system identification," Proceedings of the 11th IEEE Signal Processing Workshop on Statistical Signal Processing, 6-8 Aug. pp.341-344, 2001.

[17] Debiasi, M., and Samimy, M., "An Experimental Study of the Cavity Flow for Closed-Loop Flow Control,” AIAA Paper No: 2003-4003, June 2003.

[18] Debiasi, M. and Samimy, M., "Logic-Based Active Control of Subsonic Cavity Flow Resonance," AIAA Journal, Vol. 42, No. 9, pp. 19011909, September 2004.

[19] Efe, M.Ö., Debiasi, M., Özbay, H., and Samimy, M., "Modeling of Subsonic Cavity Flows by Neural Networks," International Conference on Mechatronics (ICM'04), June 3-5, Istanbul, Turkey, pp.560-565, 2004.

[20] Efe, M.Ö., Debiasi, M., Yan, P., Özbay, H. and Samimy, M., "Control of Subsonic Cavity Flows by Neural Networks - Analytical Models and Experimental Validation," 43rd AIAA Aerospace Sciences Meeting and Exhibit, January 10-13, 2005, Reno, Nevada, U.S.A. (Paper No: AIAA2005-0294)

[21] Yan, P., Debiasi, M., Yuan, X., Caraballo, E., Efe, M.Ö., Özbay, H., Samimy, M., DeBonis, J., Camphouse, R.C., Myatt, J. H., Serrani, A., and Malone, J., "Controller Design for Active Closed-Loop Control of Cavity Flows”, AIAA Paper No: 2004-0573, January 2004. 